\title{
Holonomy Groups of Stable Vector Bundles
}

\author{
By \\ V. BALAJI* and János KollÁR**
}

\begin{abstract}
We define the notion of holonomy group for a stable vector bundle $F$ on a variety in terms of the Narasimhan-Seshadri unitary representation of its restriction to curves.

Next we relate the holonomy group to the minimal structure group and to the decomposition of tensor powers of $F$. Finally we illustrate the principle that either the holonomy is large or there is a clear geometric reason why it should be small.
\end{abstract}

Let $M$ be a Riemannian manifold and $E$ a vector bundle with a connection $\nabla$. Parallel transport along loops gives a representation of the loop group of $M$ with base point $x$ into the orthogonal group $O\left(E_{x}\right)$ of the fiber at $x$ (see, for instance, [KN96], [Bry00]).

If $X$ is a complex manifold and $E$ a holomorphic vector bundle, then usually there are no holomorphic connections on $E$. One can, nonetheless, define a close analog of the holonomy representation in the complex setting if $E$ is a stable vector bundle and $X$ is projective algebraic.

By Mehta-Ramanathan [MR82], if $x \in C \subset X$ is a sufficiently general complex curve, then $\left.E\right|_{C}$ is also stable and so, by a result of NarasimhanSeshadri [NS65], it corresponds to a unique unitary representation $\rho: \pi_{1}(C) \rightarrow$ $U\left(E_{x}\right)$ if $c_{1}\left(\left.E\right|_{C}\right)=0$. If $c_{1}\left(\left.E\right|_{C}\right) \neq 0$, one gets a special type of unitary representation $\rho: \pi_{1}(C \backslash x) \rightarrow U\left(E_{x}\right)$, see (12). We call these the NarasimhanSeshadri representation of $\left.E\right|_{C}$.

\footnotetext{
Communicated by S. Mukai. Recieved July 10, 2006. Revised March 16, 2007.

2000 Mathematics Subject Classification(s): Primary 14J60, 32L05; Secondary 14F05, $53 \mathrm{C} 29$.

Key words: vector bundle, stability, holonomy group, parabolic bundle.

*Chennai Math. Inst. SIPCOT IT Park, Siruseri-603103, India.

e-mail: balaji@cmi.ac.in

**Princeton University, Princeton NJ 08544-1000, USA.

e-mail: kollar@math.princeton.edu
}

(C) 2008 Research Institute for Mathematical Sciences, Kyoto University. All rights reserved. 
The image of the representation, and even the Hermitian form on $E_{x}$ implicit in its definition, depend on the choice of $C$, but the picture stabilizes if we look at the Zariski closure of the image in $G L\left(E_{x}\right)$. The resulting group can also be characterized in different ways.

Theorem 1. Let $X$ be a smooth projective variety, $H$ an ample divisor on $X, E$ a stable vector bundle and $x \in X$ a point. Then there is a unique reductive subgroup $H_{x}(E) \subset G L\left(E_{x}\right)$, called the holonomy group of $E$, characterized by either of the two properties:

(1) $H_{x}(E) \subset G L\left(E_{x}\right)$ is the smallest algebraic subgroup satisfying the following:

For every curve $x \in C \subset X$ such that $\left.E\right|_{C}$ is stable, the image of the Narasimhan-Seshadri representation is contained in $H_{x}(E)$.

(2) If $C$ is sufficiently general, then the image of the Narasimhan-Seshadri representation is Zariski dense in $H_{x}(E)$.

Furthermore:

(3) For every $m, n$, the fiber map $F \rightarrow F_{x}$ gives a one-to-one correspondence between direct summands of $E^{\otimes m} \otimes\left(E^{*}\right)^{\otimes n}$ and $H_{x}(E)$-invariant subspaces of $E_{x}^{\otimes m} \otimes\left(E_{x}^{*}\right)^{\otimes n}$.

(4) The conjugacy class of $H_{x}(E)$ is the smallest reductive conjugacy class $G$ such that the structure group of $E$ can be reduced to $G$.

Remark 2. (1) The existence of a smallest reductive structure group is established in [Bog94, Thm.2.1].

(2) We emphasize that the holonomy group is defined as a subgroup of $G L\left(E_{x}\right)$ and not just as a conjugacy class of subgroups.

(3) It follows from (1.1) that the holonomy group does not depend on $H$. Thus the definition of the holonomy group makes sense for any vector bundle that is stable with respect to some ample divisor $H$.

(4) The property (1.3) almost characterizes the holonomy group. The only remaining ambiguity comes from the center of $G L\left(E_{x}\right)$. In general, the holonomy group is determined by knowing, for every $m, n \geq 0$, the direct summands of $E^{\otimes m} \otimes\left(E^{*}\right)^{\otimes n}$ and also knowing which rank 1 summands are isomorphic to $\mathcal{O}_{X}$.

(5) The above theorem has immediate generalizations to the case when $X$ is a normal variety, $E$ a reflexive sheaf with arbitrary $\operatorname{det} E$ or a sheaf with 
parabolic structure. These are discussed in (20) and (38). The case of Higgs bundles will be considered elsewhere.

(6) For some closely related ideas and applications to the construction of stable principal bundles on surfaces, see [Bal05].

Our next aim is to study and use holonomy groups by relying on the following:

Principle 3. Let $E$ be a stable vector bundle on a smooth projective variety $X$.

(1) If $E$ is "general" then the holonomy group $H_{x}(E)$ is "large", meaning, for instance, that $H_{x}(E) \supset S L\left(E_{x}\right)$.

(2) Otherwise there is geometric reason why $H_{x}(E)$ is small.

Let $\rho: \pi_{1}(X) \rightarrow U(V)$ be an irreducible representation with finite image $G$ and $E_{\rho}$ the corresponding flat vector bundle on $X$. Then $H_{x}\left(E_{\rho}\right)=G$. Understanding $G$ in terms of its representations $V^{\otimes m}$ is certainly possible, but it quickly leads to intricate questions of finite group theory. (See [GT05] for such an example.) There is a significant case when we can avoid the complications coming from finite subgroups of $G L(V)$.

Proposition 4. If $X$ is simply connected then $H_{x}(E)$ is connected.

The representation theory of connected reductive groups is quite well understood, and this enables us to get some illustration of the above Principle.

Proposition 5. Let $E$ be a stable vector bundle on a simply connected smooth projective variety $X$. Then the following are equivalent:

(1) $S^{m} E$ is stable (that is, indecomposable) for some $m \geq 2$.

(2) $S^{m} E$ is stable (that is, indecomposable) for every $m \geq 2$.

(3) The holonomy is one of the following:

(a) $S L\left(E_{x}\right)$ or $G L\left(E_{x}\right)$,

(b) $S p\left(E_{x}\right)$ or $G S p\left(E_{x}\right)$ for a suitable nondegenerate symplectic form on $E_{x}$ (and $\operatorname{rank} E$ is even).

Note that the statements (5.1) and (5.2) do not involve the holonomy group, but it is not clear to us how to prove their equivalence without using holonomy.

If $X$ is not simply connected, the results of [GT06] imply the following: 
Corollary 6. Let $E$ be a stable vector bundle on a smooth projective variety $X$ of rank $\neq 2,6,12$. Then the following are equivalent:

(1) $S^{r} E$ is stable for some $r \geq 4$.

(2) $S^{r} E$ is stable for every $r \geq 2$.

(3) The commutator of the holonomy group is either $S L\left(E_{x}\right)$ or $S p\left(E_{x}\right)$.

The exceptional cases in ranks 2,6,12 are classified in [GT06, Thm.1.1]. They are connected with the simple groups $A_{5}, J_{2}, G_{2}(4)$ and $S u z$. Even in these cases, the equivalence between (6.1-2) holds if the assumption $r \geq 4$ is replaced by $r \geq 6$.

Another illustration of the Principle (3) is the following partial description of low rank bundles.

Proposition 7. Let $E$ be a stable vector bundle on a simply connected smooth projective variety $X$. Assume that $\operatorname{det} E \cong \mathcal{O}_{X}$ and $\operatorname{rank} E \leq 7$. Then one of the following holds.

(1) The holonomy group is $S L\left(E_{x}\right)$.

(2) The holonomy is contained in $S O\left(E_{x}\right)$ or $S p\left(E_{x}\right)$. In particular, $E \cong$ $E^{*}$ and the odd Chern classes of $E$ are 2-torsion.

(3) $E$ is obtained from a rank 2 vector bundle $F_{2}$ and a rank 3 vector bundle $F_{3}$. There are 2 such cases which are neither orthogonal nor symplectic:
(a) $\operatorname{rank} E=6$ and $E \cong S^{2} F_{3}$, or
(b) $\operatorname{rank} E=6$ and $E \cong F_{2} \otimes F_{3}$.

There are two reasons why a result of this type gets more complicated for higher rank bundles.

First, already in rank 7 , we have vector bundles with $G_{2}$ holonomy. It is not on our list separately since $G_{2} \subset S O_{7}$. It is quite likely that there is some very nice geometry associated with $G_{2}$ holonomy, but this remains to be discovered. Similarly, the other exceptional groups must all appear for the higher rank cases.

Second, and this is more serious, there are many cases where the holonomy group is not simply connected, for instance $P G L$. In this case there is a Brauer obstruction to lift the structure group to $G L$ and to write $E$ in terms of a lower 
rank bundle using representation theory. We study this in (44). In the low rank cases we are saved by the accident that such representations happen to be either orthogonal or symplectic, but this definitely fails in general.

8 (Comparison with the differential geometric holonomy). For the tangent bundle of a smooth projective variety $X$, one gets two notions of holonomy. The classical differential geometric holonomy and the algebraic holonomy defined earlier. These are related in some ways, but the precise relationship is still unclear.

First of all, the algebraic holonomy makes sense whenever $T_{X}$ is stable, and it does not depend on the choice of a metric on $X$. The differential geometric holonomy depends on the metric chosen.

If $X$ admits a Kähler-Einstein metric, then its holonomy group, which is a subgroup of the unitary group $U\left(T_{x} X\right)$, is canonically associated to $X$.

By contrast, the algebraic holonomy is not unitary. For a general curve $C \subset X$, the Narasimhan-Seshadri representation gives a subgroup of a unitary group, but the Hermitian form defining the unitary group in question does depend on $C$, except when $X$ is a quotient of an Abelian variety.

Thus the processes that define the holonomy group in algebraic geometry and in differential geometry are quite different. It is, nonetheless, possible, that the two holonomy groups are closely related.

Question 9. Let $X$ be a simply connected smooth projective variety which has a Kähler-Einstein metric. Is the algebraic holonomy group of $T_{X}$ the complexification of the differential geometric holonomy group? ${ }^{1}$

For non simply connected varieties the differential geometric holonomy group may have infinitely many connected components, and one may need to take the complexification of its Zariski closure instead.

It is possible that (9) holds for the simple reason that the algebraic holonomy group of a tangent bundle is almost always $G L_{n}$. The differential geometric holonomy group is almost always $U_{n}$, with two notable exceptions. In both of them, the answer to (9) is positive.

Proposition 10 (Calabi-Yau varieties). Let $X$ be a simply connected smooth projective variety $X$ such that $K_{X}=0$ which is not a direct product.

The differential geometric holonomy group is either $S U_{n}(\mathbb{C})$ or $U_{n}(\mathbb{H})$. Correspondingly, the algebraic holonomy group is $S L_{n}(\mathbb{C})\left(\right.$ resp. $\left.S p_{2 n}(\mathbb{C})\right)$.

\footnotetext{
${ }^{1}$ This was recently settled by Biswas, see [Bis07].
} 
Proposition 11 (Homogeneous spaces). Let $X=G / P$ be a homogeneous space such that the stabilizer representation of $P$ on $T_{x} X$ is irreducible. Then $T_{X}$ is stable and the algebraic holonomy is the image of this stabilizer representation.

\section{$\S 1$. Variation of Monodromy Groups}

12. Let $C$ be a smooth projective curve over $\mathbb{C}$ and $x \in C$ a point. Every unitary representation $\rho: \pi_{1}(C, x) \rightarrow U\left(\mathbb{C}^{r}\right)$ gives a flat vector bundle $E_{\rho}$ of rank $r$. By [NS65], this gives a real analytic one-to-one correspondence between conjugacy classes of unitary representations and polystable vector bundles of rank $r$ and degree 0 .

The similar correspondence between representations and polystable vector bundles of rank $r$ and degree $d \neq 0$ is less natural and it depends on an additional point of $C$.

Let $C$ be a smooth projective curve over $\mathbb{C}$ and $x \neq c \in C$ two points. Let $\Gamma \subset \pi_{1}(C \backslash c, x)$ denote the conjugacy class consisting of counterclockwise lassos around $c$.

A unitary representation

$$
\rho: \pi_{1}(C \backslash c, x) \rightarrow U\left(\mathbb{C}^{r}\right) \text { such that } \rho(\gamma)=e^{2 \pi i d / r} \mathbf{1}
$$

for every $\gamma \in \Gamma$ is said to have type $d / r$. (In the original definition this is called type $d$. Using type $d / r$ has the advantage that irreducible subrepresentations have the same type.) Note that the type is well defined only modulo 1.

By [NS65], for every $r$ and $d$ the following hold:

(1) There is a one-to-one correspondence

$$
N S:(C, c, x, E) \mapsto\left[\rho: \pi_{1}(C \backslash c, x) \rightarrow U\left(E_{x}\right)\right]
$$

between

(a) polystable vector bundles $E$ of rank $r$ and degree $d$ over a smooth projective curve $C$ with 2 marked points $x, c$, and

(b) isomorphism classes of unitary representations $\rho: \pi_{1}(C \backslash c, x) \rightarrow$ $U\left(\mathbb{C}^{r}\right)$ of type $d / r$.

(2) NS depends real analytically on $(C, c, x, E)$.

(3) The fiber map $F \rightarrow F_{x}$ gives a one-to-one correspondence between 
(a) direct summands of $E^{\otimes m} \otimes\left(E^{*}\right)^{\otimes n}$, and

(b) $\pi_{1}(C \backslash c, x)$ invariant subspaces of $E_{x}^{\otimes m} \otimes\left(E_{x}^{*}\right)^{\otimes n}$.

(This is stated in [NS65] for $0 \leq d<r$. In the general case, we twist $E$ by a suitable $\mathcal{O}_{C}(m c)$ and then apply [NS65].)

Because of the artificial role of the point $c$, one has to be careful in taking determinants. The representation $\operatorname{det} \rho$ corresponds to the degree 0 line bundle $\mathcal{O}_{C}(-d[c]) \otimes \operatorname{det} E$.

Definition 13. Let $E$ be a stable vector bundle on a smooth projective curve $C$ and $x, c \in C$ closed points. The Zariski closure of the image of the Narasimhan-Seshadri representation $\rho_{c}: \pi_{1}(C \backslash c, x) \rightarrow G L\left(E_{x}\right)$ is called the algebraic monodromy group of $E$ at $(C, x, c)$ and it is denoted by $M_{x}(E, C, c)$. Note that $M_{x}(E, C, c)$ is reductive since it is the Zariski closure of a subgroup of a unitary group.

$M_{x}(E, C, c)$ depends on the point $c$ but only slightly. Choosing a different $c$ corresponds to tensoring $E$ with a different line bundle, which changes the representation by a character $\pi_{1}(C \backslash c, x) \rightarrow \mathbb{C}^{*}$.

As we see in (15), for very general $c \in C$ we get the same $M_{x}(E, C, c)$. We denote this common group by $M_{x}(E, C)$.

Lemma 14. If $\operatorname{det} E$ is torsion in $\operatorname{Pic} C$ then the image of $\operatorname{det}: M_{x}(E$, $C) \rightarrow \mathbb{C}^{*}$ is torsion. Otherwise $M_{x}(E, C)$ contains the scalars $\mathbb{C}^{*} \subset G L\left(E_{x}\right)$.

Proof. As we noted above,

$$
E_{\operatorname{det} \rho_{c}} \cong \mathcal{O}_{C}(-(\operatorname{deg} E)[c]) \otimes \operatorname{det} E .
$$

If $\operatorname{deg} E \neq 0$, then $E_{\operatorname{det} \rho_{c}}$ is a nonconstant family of degree zero line bundles on $C$, hence its general member is not torsion in $\operatorname{Pic} C$. Thus in this case det : $M_{x}(E, C) \rightarrow \mathbb{C}^{*}$ is surjective.

If $\operatorname{deg} E=0$ then $E_{\operatorname{det} \rho_{c}} \cong \operatorname{det} E$ is constant. Thus $\operatorname{det}: M_{x}(E, C) \rightarrow \mathbb{C}^{*}$ is surjective iff $\operatorname{det} E$ is not torsion in $\operatorname{Pic} C$.

Since $M_{x}(E, C)$ is reductive, we see that det $: M_{x}(E, C) \rightarrow \mathbb{C}^{*}$ is surjective iff the center of $M_{x}(E, C)$ is positive dimensional.

If $E$ is stable then $M_{x}(E, C, c)$ acts irreducibly on $E_{x}$, and so the center consists of scalars only. Thus we conclude that if $\operatorname{det} E$ is not torsion in $\operatorname{Pic}(C)$ then the scalars are contained in $M_{x}(E, C)$. In general, it is easy to see that $M_{x}(E, C)=\mathbb{C}^{*} \cdot M_{x}(E, C, c)$ for any $c \in C \backslash x$ if $\operatorname{det} E$ is not torsion in $\operatorname{Pic}(C)$. 
The Narasimhan-Seshadri representations $\rho$ vary real analytically with $(C, c, x, E)$ but the variation is definitely not complex analytic. So it is not even clear that the groups $M_{x}(E, C)$ should vary algebraically in any sense. Nonetheless, the situation turns out to be quite reasonable.

Lemma 15. Let $g: U \rightarrow V$ be a flat family of smooth projective curves with sections $s_{x}, s_{c}: V \rightarrow U$. Let $E \rightarrow U$ be a vector bundle of rank $r$ such that $\left.E\right|_{U_{v}}$ is polystable for every $v \in V$. For every $v \in V$ let

$$
\rho_{v}: \pi_{1}\left(U_{v} \backslash s_{c}(v), s_{x}(v)\right) \rightarrow U\left(E_{s_{x}(v)}\right)
$$

be the corresponding Narasimhan-Seshadri representation and let $M_{v} \subset$ $G L\left(E_{s_{x}(v)}\right)$ be the Zariski closure of its image.

Then there is an open set $V^{0} \subset V$ and a flat, reductive group scheme $G \subset G L\left(s_{x}^{*} E\right) \rightarrow V^{0}$ such that $M_{v}=G_{v}$ for very general $v \in V^{0}$. (That is, for all $v$ in the complement of countably many subvarieties of $V^{0}$.)

Remark 16. By [Ric72, 3.1], the fibers of a flat, reductive group scheme are conjugate to each other. The conjugacy class of the fibers $G_{v} \subset G L\left(\mathbb{C}^{r}\right)$ is called the generic monodromy group of $E$ on $U / V$. Note that while the monodromy groups $M_{x}(E, C)$ are subgroups of $G L\left(E_{x}\right)$, the generic monodromy group is only a conjugacy class of subgroups.

In most cases $M_{v}=G_{v}$ for every $v \in V^{0}$, but there are many exceptions. The simplest case is when $V=C$ is an elliptic curve, $U=C \times C$ and $E$ is the universal degree 0 line bundle.

Then $M_{c}=\mathbb{C}^{*}$ if $c \in C$ is not torsion in $C$ but $M_{c}=\mu_{n}$, the groups of $n$th roots of unity, if $c \in C$ is $n$-torsion.

17 (Proof of $(15))$. Let $W$ be a vector space of dimension $r$. The general orbit of $G L(W)$ on $\left(W^{r}+\operatorname{det}^{-1} W\right)^{*}$ is closed, hence the same holds for any closed subgroup of $G L(W)$. We can thus recover the stable orbits of $G$, and hence $G$ itself, as the general fibers of the rational map

$$
h_{W}:\left(W^{r}+\operatorname{det}^{-1} W\right)^{*} \rightarrow \operatorname{Spec} \sum_{m \geq 0}\left(S^{m r}\left(W^{r}\right) \otimes \operatorname{det}^{-m} W\right)^{G} .
$$

Correspondingly, if $E \rightarrow C$ is a rank $r$ vector bundle corresponding to a unitary representation $\rho: \pi_{1}(C \backslash c, x) \rightarrow U\left(E_{x}\right)$, then we can recover the Zariski closure of $\operatorname{im} \rho$ from the general fibers of the rational map

$$
\begin{aligned}
h_{C}:\left(E_{x}^{r}+\operatorname{det}^{-1} E_{x}\right)^{*}-\rightarrow & \operatorname{Spec} \sum_{m \geq 0}\left(S^{m r}\left(E_{x}^{r}\right) \otimes \operatorname{det}^{-m} E_{x}\right)^{G} \\
\downarrow & \cong \\
& \quad \operatorname{Spec} \sum_{m \geq 0} H^{0}\left(C, S^{m r}\left(E^{r}\right) \otimes \operatorname{det}^{-m} E\right) .
\end{aligned}
$$


Let us now apply this to our family $g: U \rightarrow V$. Then we get a rational map

$$
h_{V}:\left(s_{x}^{*} E^{r}+\operatorname{det}^{-1} s_{x}^{*} E\right)^{*} \rightarrow \sum_{m \geq 0} g_{*}\left(S^{m r}\left(E^{r}\right) \otimes \operatorname{det}^{-m} E\right) .
$$

Each of the sheaves

$$
g_{*}\left(S^{m r}\left(E^{r}\right) \otimes \operatorname{det}^{-m} E\right)
$$

commutes with base change over an open set $V_{m} \subset V$, but these open sets may depend on $m$. By the above remarks, for every point $v \in \cap_{m \geq 1} V_{m}$, a general fiber of $h_{V}$ above $v$ is the Zariski closure of the unitary representation $\rho_{v}$.

Over the generic point $v_{\text {gen }} \in V$ we get a reductive group scheme $G_{\text {gen }} \subset$ $G L\left(E_{s\left(v_{g e n}\right)}\right)$ which extends to a reductive group scheme $G \subset G L\left(\left.s^{*} E\right|_{V^{0}}\right) \rightarrow$ $V^{0}$ over a suitable open set $V^{0}$.

The very general points in the lemma will be, by definition, the points in the intersection $\cap_{m \geq 0} V_{m}$.

By taking the closure of $G$ in $G L\left(s^{*} E\right)$, we obtain an open subset $V^{*} \subset V$ such that

(1) the closure of $G$ in $G L\left(\left.s^{*} E\right|_{V^{*}}\right)$ is a flat group scheme (but possibly not reductive), and

(2) $V \backslash V^{*}$ has codimension $\geq 2$ in $V$.

Lemma 18. Notation as above. For every $v \in V^{*}$,

(1) $M_{v} \subset G_{v}^{*}$

(2) $M_{v}$ is conjugate to a subgroup of the generic monodromy group, and

(3) if $\operatorname{dim} M_{v}=\operatorname{dim} G_{v}^{*}$ then in fact $M_{v}=G_{v}^{*}$.

Proof. $U \rightarrow V$ is topologically a product in a Euclidean neighborhood of $v \in W \subset V^{*}$, thus we can think of the family of representations $\rho_{v}$ as a continuous map

$$
\rho: W \times \pi_{1}\left(U_{v} \backslash c(v), x(v)\right) \rightarrow G L\left(\mathbb{C}^{r}\right)
$$

By (15), for very general $w \in W, \rho\left(\{w\} \times \pi_{1}\left(U_{v} \backslash c(v), x(v)\right) \subset G_{w}^{*}\right.$, hence, by continuity, $\rho\left(\{v\} \times \pi_{1}\left(U_{v} \backslash c(v), x(v)\right) \subset G_{v}^{*}\right.$, which proves (1).

Since $M_{v}$ is reductive, by [Ric72,3.1], it is conjugate to a subgroup of $G_{w}^{*}$ for $w$ near $v$, hence to a subgroup of the generic monodromy group. 
Finally, if $\operatorname{dim} M_{v}=\operatorname{dim} G_{v}^{*}$, then the connected component of $G_{v}^{*}$ is the same as the connected component of $M_{v}$, hence $G_{v}^{*}$ is reductive and again by [Ric72, 3.1], it is conjugate to a subgroup of the generic monodromy group. Since $\rho\left(\{w\} \times \pi_{1}\left(U_{v} \backslash c(v), x(v)\right)\right.$ has points in every connected component of $G_{w}^{*}$, by continuity the same holds for $\rho\left(\{v\} \times \pi_{1}\left(U_{v} \backslash c(v), x(v)\right)\right.$. Thus in fact $M_{v}=G_{v}^{*}$.

\section{§2. Holonomy Groups}

Let $X$ be a normal, projective variety of dimension $d$ with an ample divisor $H$. A curve $C \subset X$ is called a complete intersection (or CI) curve of type $\left(a_{1}, \ldots, a_{d-1}\right)$ if $C$ is a (scheme theoretic) intersection of $(d-1)$ divisors $D_{i} \in$ $\left|a_{i} H\right|$. We say that $C \subset X$ is a general CI curve of type $\left(a_{1}, \ldots, a_{d-1}\right)$ if the divisors $D_{i} \in\left|a_{i} H\right|$ are all general.

If a smooth point $x \in X$ is fixed then a general CI curve of type $\left(a_{1}, \ldots\right.$, $\left.a_{d-1}\right)$ through $x$ is an intersection of $(d-1)$ general divisors $D_{i} \in\left|a_{i} H\right|$, each passing through $x$.

Let $E$ be a reflexive sheaf on $X$ such that $E$ is $\mu$-stable with respect to $H$. By [MR82] this is equivalent to assuming that $\left.E\right|_{C}$ is a stable vector bundle for a general CI curve $C$ of type $\left(a_{1}, \ldots, a_{d-1}\right)$ for $a_{i} \gg 1$.

If $E$ is locally free at the points $x_{1}, \ldots, x_{s}$, then this is also equivalent to assuming that $\left.E\right|_{C}$ is a stable vector bundle for a general CI curve $C$ of type $\left(a_{1}, \ldots, a_{d-1}\right)$ passing through the points $x_{1}, \ldots, x_{s}$ for $a_{i} \gg 1$. (While this stronger form of [MR82] is not stated in the literature, it is easy to modify the proofs to cover this more general case.)

Definition 19. Let $X$ be a normal, projective variety of dimension $n$ with an ample divisor $H$ and $E$ a reflexive sheaf on $X$ such that $E$ is $\mu$-stable with respect to $H$. Assume that $E$ is locally free at $x$.

Let $B \subset X$ be the set of points where either $X$ is singular or $E$ is not locally free. Then $B$ has codimension at least 2 in $X$. This implies that all general CI curves are contained in $X \backslash B$ and there is a one-to-one correspondence between saturated subsheaves of the reflexive hull of $E^{\otimes m} \otimes\left(E^{*}\right)^{\otimes n}$ and saturated subsheaves of $\left.E^{\otimes m} \otimes\left(E^{*}\right)^{\otimes n}\right|_{X \backslash B}$.

The holonomy group of $E$ at $x$ is the unique smallest subgroup $H_{x}(E) \subset$ $G L\left(E_{x}\right)$ such that:

For every smooth, pointed, projective curve $(D, d, y)$ and every morphism $g: D \rightarrow X$ such that $g(y)=x, E$ is locally free along $g(D)$ and $g^{*} E$ is polystable, the image of the Narasimhan-Seshadri representation of $\pi_{1}(D \backslash d, y)$ is contained in $H_{x}(E) \subset G L\left(E_{x}\right)=G L\left(\left(g^{*} E\right)_{y}\right)$. 
Theorem 20. Notation and assumptions as in (19).

(1) Let $C$ be a very general $C I$ curve of type $\left(a_{1}, \ldots, a_{d-1}\right)$ through $x$ for $a_{i} \gg 1$. Then the image of the Narasimhan-Seshadri representation of $\pi_{1}(C \backslash c, x)$ is Zariski dense in $H_{x}(E)$. In particular, $H_{x}(E)$ is reductive.

(2) For every $m, n$, the fiber map $F \rightarrow F_{x}$ gives a one-to-one correspondence between direct summands of the reflexive hull of $E^{\otimes m} \otimes\left(E^{*}\right)^{\otimes n}$ and $H_{x}(E)$ invariant subspaces of $E_{x}^{\otimes m} \otimes\left(E_{x}^{*}\right)^{\otimes n}$.

(3) The conjugacy class of $H_{x}(E)$ is the smallest reductive conjugacy class $G$ such that the structure group of $E$ can be reduced to $G$.

Remark 21. For every curve $C$, the image of the unitary representation of $\pi_{1}(C \backslash c, x)$ is contained in a maximal compact subgroup of $H_{x}(E)$. While $H_{x}(E)$ is well defined as a subgroup of $G L\left(E_{x}\right)$, we do not claim that this maximal compact subgroup of $H_{x}(E)$ is independent of $C$. Most likely the opposite holds: the maximal compact subgroup is independent of $C$ iff $E$ is a flat vector bundle on $X \backslash \operatorname{Sing} X$.

22 (Proof of $(20))$. Fix $\left(a_{1}, \ldots, a_{d-1}\right)$ such that $\left.E\right|_{C}$ is stable for a general CI curve $C$ of type $\left(a_{1}, \ldots, a_{d-1}\right)$. By (15), the conjugacy class of $M_{x}(E, C$, c) $\subset G L\left(E_{x}\right)$ is independent of $C$ for very general $C$ of type $\left(a_{1}, \ldots, a_{d-1}\right)$ and $c \in C$. Denote this conjugacy class by $M_{x}\left(a_{1}, \ldots, a_{d-1}\right)$. First we show that these conjugacy classes $M_{x}\left(a_{1}, \ldots, a_{d-1}\right)$ stabilize.

Lemma 23. There is a conjugacy class $M_{x}$ of subgroups of $G L\left(E_{x}\right)$ such that if the $a_{i}$ are sufficiently divisible then $M_{x}\left(a_{1}, \ldots, a_{d-1}\right)=M_{x}$.

Proof. Fix a very general CI curve $C$ of type $\left(a_{1}, \ldots, a_{d-1}\right)$ such that $\left.E\right|_{C}$ is stable. We compare the monodromy group $M_{x}(E, C, c)$ with the monodromy group $M_{x}\left(E, C_{k}, c_{k}\right)$ where $C_{k}$ is a very general CI curve of type $k a_{1}, a_{2}, \ldots, a_{d-1}$.

The divisors $D_{2}, \ldots, D_{d-1}$ do not need changing, so we may assume that $\operatorname{dim} X=2$. Then $C$ is defined by a section $u \in H^{0}\left(X, \mathcal{O}_{X}\left(a_{1} H\right)\right)$. Choose a general $v \in H^{0}\left(X, \mathcal{O}_{X}\left(k a_{1} H\right)\right)$ vanishing at $x$ and consider the family of curves $C_{t}^{\prime}:=\left(u^{k}+t^{k} v=0\right)$. The general member is a CI curve $C_{t}^{\prime}$ of type $\left(k a_{1}, a_{2}, \ldots, a_{d-1}\right)$ through $x$.

Note that Supp $C_{0}^{\prime}=C$ but $C_{0}^{\prime}$ has multiplicity $k$ along $C$. The family is not normal along $C_{0}$ and we can normalize it by introducing the new variable 
$u / t$. We then get a family of curves $C_{t}$ such that $C_{t}=C_{t}^{\prime}$ for $t \neq 0$ and $C_{0}$ is a smooth curve, which is a degree $k$ cyclic cover $g: C_{0} \rightarrow C$ ramified at the intersection points $(u=v=0)$.

Since $C_{0} \rightarrow C$ is totally ramified at $x$, we see that $g_{*}: \pi_{1}\left(C_{0} \backslash c_{0}, x\right) \rightarrow$ $\pi_{1}(C \backslash c, x)$ is surjective where $c_{0} \in C_{0}$ is any preimage of $c$. In particular,

$$
M_{x}\left(g^{*} E, C_{0}, c_{0}\right)=M_{x}(E, C, c) .
$$

We can apply (18) to the family $\left\{C_{t}\right\}$ to conclude that

$$
\operatorname{dim} M_{x}\left(a_{1}, \ldots, a_{d-1}\right) \leq \operatorname{dim} M_{x}\left(k a_{1}, \ldots, a_{d-1}\right),
$$

and if equality holds then $M_{x}\left(a_{1}, \ldots, a_{d-1}\right)$ and $M_{x}\left(k a_{1}, \ldots, a_{d-1}\right)$ are conjugate.

Thus if we choose $\left(a_{1}, \ldots, a_{d-1}\right)$ such that $\operatorname{dim} M_{x}\left(a_{1}, \ldots, a_{d-1}\right)$ is maximal, then $M_{x}\left(a_{1}, \ldots, a_{d-1}\right)$ and $M_{x}\left(b_{1}, \ldots, b_{d-1}\right)$ are conjugate whenever $a_{i} \mid b_{i}$ for every $i$.

Choose $\left(a_{1}, \ldots, a_{d-1}\right)$ and a very general CI curve of type $\left(a_{1}, \ldots, a_{d-1}\right)$ through $x$ such that

(1) $M_{x}\left(a_{1}, \ldots, a_{d-1}\right)=M_{x}$, and

(2) every stable summand of $T(E)$ restricts to a stable bundle on $C$.

Claim 24. With the above notation, $H_{x}(E)=M_{x}$.

Proof. $M_{x} \subset H_{x}(E)$ by definition.

By assumption $M_{x}$ is the stabilizer of a nonzero vector $w_{x} \in T(E)_{x}=$ $E_{x}^{\otimes m} \otimes\left(E_{x}^{*}\right)^{\otimes n}$, thus it corresponds to a direct summand $\left.\mathcal{O}_{C} \cong W_{C} \hookrightarrow T(E)\right|_{C}$ which in turn gives a direct summand $\mathcal{O}_{X} \cong W_{X} \hookrightarrow T(E)$ by the second assumption.

Pick any smooth pointed curve $(D, d, y)$ and a map $g: D \rightarrow X \backslash B$ such that $g(y)=x$ and $g^{*} E$ is polystable. Then $\mathcal{O}_{D} \cong g^{*} W \subset g^{*}(T(E))$ is a direct summand, hence the Narasimhan-Seshadri representation of $\pi_{1}(D \backslash d, y)$ in $g^{*}\left(T\left(E_{x}\right)\right)=T\left(g^{*} E_{y}\right)$ fixes $w$. The stabilizer of $w$ is exactly $M_{x}$, hence the monodromy group of $g^{*} E$ is contained in $M_{x}$. Since this holds for any $(D, d, y)$, we see that $H_{x}(E)=M_{x}$.

Claim 25. The stabilizer of $\left.W\right|_{X \backslash B} \hookrightarrow T\left(\left.E\right|_{X \backslash B}\right)$ in $G L\left(\left.E\right|_{X \backslash B}\right)$ is a reductive subgroup scheme $H \subset G L\left(\left.E\right|_{X \backslash B}\right)$ whose fibers are in the conjugacy class $M_{x}$. The structure group of $\left.E\right|_{X \backslash B}$ can be reduced to a conjugacy class $G$ iff some group in $G$ contains $M_{x}$. 
Proof. By construction $H_{x}=M_{x}$ is reductive, hence there is a largest open set $X^{0} \subset X$ such that the fibers $H_{v}$ are in the conjugacy class $M_{x}$ for every $v \in X^{0}$. Thus the structure group of $\left.E\right|_{X^{0}}$ can be reduced to $M_{x}$.

Pick a very general CI curve $C$ of type $\left(a_{1}, \ldots, a_{d-1}\right)$ such that $\left.E\right|_{C}$ is stable and $M_{x}(E, C)=M_{x}$. The stabilizer of every point of $\left.W\right|_{C}$ is conjugate to $M_{x}(E, C)$, which shows that $X^{0}$ contains $C$. This implies that $X \backslash X^{0}$ has codimension $\geq 2$ in $X$.

By Hartogs' theorem, a rational map from a normal variety to an affine variety which is defined outside a codimension two set is everywhere defined, thus the structure group of $\left.E\right|_{X \backslash B}$ also reduces to $M_{x}$.

Conversely, if the structure group of $E$ can be reduced to the conjugacy class $G \subset G L(E)$, then the structure group of $\left.E\right|_{C}$ can also be reduced to $G$, hence some group in the conjugacy class $G$ contains $M_{x}$.

It remains to show that (20.1) holds for $\left(a_{1}, \ldots, a_{d-1}\right)$ sufficiently large. (So far we have established (20.1) only for $\left(a_{1}, \ldots, a_{d-1}\right)$ sufficiently divisible.)

Fix now $\left(a_{1}, \ldots, a_{d-1}\right)$ such that $M_{x}\left(a_{1}, \ldots, a_{d-1}\right)=M_{x}$. We claim that in fact $M_{x}\left(b_{1}, \ldots, b_{d-1}\right)=M_{x}$ for every $b_{i} \geq 2 a_{i}$.

Indeed, assume the contrary. By (18) we know that $M_{x}\left(b_{1}, \ldots, b_{d-1}\right)$ is conjugate to a subgroup of $M_{x}$. Thus if they are not equal, then there are $m, n$ and a vector $v \in E_{x}^{\otimes m} \otimes\left(E_{x}^{*}\right)^{\otimes n}$ which is stabilized by $M_{x}\left(b_{1}, \ldots, b_{d-1}\right)$ but not by $M_{x}$.

Correspondingly, if $D$ is a very general CI curve of type $b_{1}, \ldots, b_{d-1}$, then $v$ corresponds to a direct summand $\left.W_{D} \subset E^{\otimes m} \otimes\left(E^{*}\right)^{\otimes n}\right|_{D}$ which can not be obtained as a restriction of a direct summand of $E^{\otimes m} \otimes\left(E^{*}\right)^{\otimes n}$. Thus there is a stable direct summand $F \subset E^{\otimes m}$ such that $\left.F\right|_{D}$ is not stable. By the already proved case of (20.1), we know that $\left.F\right|_{C}$ is stable. Hence by [HL97, Lem.7.2.10], $\left.F\right|_{D}$ is also stable, a contradiction.

\section{$\S 3 . \quad$ Parabolic Bundles}

We briefly recall the correspondence between the category of parabolic bundles on $X$ and the category of $G$-bundles on a suitable cover.

Let $D$ be an effective divisor on $X$. For a coherent sheaf $E$ on $X$ the image of $E \otimes_{\mathcal{O}_{X}} \mathcal{O}_{X}(-D)$ in $E$ is denoted by $E(-D)$. The following definition of parabolic sheaves was introduced in [MY92].

Definition 26. Let $E$ be a torsion-free $\mathcal{O}_{X}$-coherent sheaf on $X$. A quasi-parabolic structure on $E$ over $D$ is a filtration by $\mathcal{O}_{X}$-coherent subsheaves

$$
E=F_{1}(E) \supset F_{2}(E) \supset \cdots \supset F_{l}(E) \supset F_{l+1}(E)=E(-D) .
$$


The integer $l$ is called the length of the filtration. A parabolic structure is a quasi-parabolic structure, as above, together with a system of weights

$$
0 \leq \alpha_{1}<\alpha_{2}<\cdots<\alpha_{l-1}<\alpha_{l}<1
$$

where the weight $\alpha_{i}$ corresponds to the subsheaf $F_{i}(E)$.

We shall denote the parabolic sheaf defined above datum by the triple $\left(E, F_{*}, \alpha_{*}\right)$. When there is no confusion it will be denoted by $E_{*}$.

For a parabolic sheaf $\left(E, F_{*}, \alpha_{*}\right)$ define the following filtration $\left\{E_{t}\right\}_{t \in \mathbb{R}}$ of coherent sheaves on $X$ parameterized by $\mathbb{R}$ :

$$
E_{t}:=F_{i}(E)(-[t] D)
$$

where $[t]$ is the integral part of $t$ and $\alpha_{i-1}<t-[t] \leq \alpha_{i}$, with the convention that $\alpha_{0}=\alpha_{l}-1$ and $\alpha_{l+1}=1$.

A homomorphism from the parabolic sheaf $\left(E, F_{*}, \alpha_{*}\right)$ to another parabolic sheaf $\left(E^{\prime}, F_{*}^{\prime}, \alpha_{*}^{\prime}\right)$ is a homomorphism from $E$ to $E^{\prime}$ which sends any subsheaf $E_{t}$ into $E_{t}^{\prime}$, where $t \in[0,1]$ and the filtration are as above.

If the underlying sheaf $E$ is locally free then $E_{*}$ will be called a parabolic vector bundle. In this section, all parabolic sheaves will be assumed to be parabolic vector bundles.

We have the following equivalent definition:

Definition 27. Let $X$ be a normal, projective variety and $D$ an effective divisor. A quasi-parabolic filtration on a sheaf $E$ is a filtration by subsheaves of the restriction $\left.E\right|_{D}$ of the sheaf $E$ to the parabolic divisor $D$ :

$$
\left.E\right|_{D}=\mathcal{F}_{D}^{1}(E) \supset \mathcal{F}_{D}^{2}(E) \supset \cdots \supset \mathcal{F}_{D}^{l}(E) \supset \mathcal{F}_{D}^{l+1}(E)=0
$$

together with a system of weights

$$
0 \leq \alpha_{1}<\alpha_{2}<\cdots<\alpha_{l-1}<\alpha_{l}<1
$$

We assume that the following conditions are satisfied:

(1) $X$ is smooth and $D$ is a divisors with normal crossings. In other words, any parabolic divisor is assumed to be reduced, its irreducible components are smooth and the irreducible components intersect transversally.

(2) All the parabolic weights are rational numbers. 
(3) On each component of the parabolic divisor the filtration is given by subbundles.

Consider the decomposition

$$
D=\sum_{i=1}^{n} D_{i} .
$$

Let $E$ be a vector bundle on $X$. For $1 \leq i \leq n$, let

$$
\left.E\right|_{D_{i}}=\mathcal{F}_{i}{ }^{1} \supset \mathcal{F}_{i}^{2} \supset \cdots \supset \mathcal{F}_{i}^{l_{i}} \supset \mathcal{F}_{i}^{l_{i}+1}=0
$$

with $l_{i} \geq 1$, be a filtration of subbundles on $D_{i}$. Suppose that we are given a string of numbers $\alpha_{j}^{i}$, with $1 \leq j \leq l_{i}+1$, satisfying the following:

$$
0 \leq \alpha_{1}^{i}<\alpha_{2}^{i}<\cdots<\alpha_{l_{i}}^{i}<\alpha_{l_{i}+1}^{i}=1 .
$$

Then we can construct a parabolic structure on $E$ as follows: Define the coherent subsheaves $\bar{F}_{i}^{j}$ of $E$, where $1 \leq j \leq l_{i}$ by the following short exact sequence:

$$
0 \rightarrow{\overline{F_{i}}}^{j} \rightarrow E \rightarrow\left(\left.E\right|_{D_{i}}\right) / \mathcal{F}_{i}{ }^{j} \rightarrow 0 .
$$

For $1 \leq i \leq n$ and $0 \leq t<1$, let

$$
l(t, i):=\min \left[j \mid j \in\left\{1, \ldots, l_{i}+1\right\} \& \alpha_{j}^{i} \geq t\right] .
$$

Define

$$
E_{t}=\cap_{i=1}^{n} \bar{F}_{i}^{l(t, i)} \subset E
$$

The filtration $\left\{E_{t}\right\}$ defines a parabolic structure on $E$ and any parabolic structure on $E$ with $D$ as parabolic divisor arises this way.

We denote the entire parabolic datum by $\left(E, F_{*}, \alpha_{*}\right)$ or simply by $E_{*}$ when the context is clear. If the underlying sheaf $E$ is locally free then $E_{*}$ is called a parabolic vector bundle.

Let $\operatorname{PVect}(X, D)$ denote the category whose objects are parabolic vector bundles over $X$ with parabolic structure over the divisor $D$ satisfying the above three conditions, and the morphisms of the category are homomorphisms of parabolic vector bundles (cf. for example [Bis97]).

The direct sum of two vector bundles with parabolic structures has an obvious parabolic structure and $\operatorname{PVect}(X, D)$ is closed under the operation of taking direct sum. We remark that the category $\operatorname{PVect}(X, D)$ is an additive tensor category with the direct sum and the parabolic tensor product operation. It is straight-forward to check that $\operatorname{PVect}(X, D)$ is also closed under the operation of taking the parabolic dual defined in [Bis97] or [Yok95]. 
For an integer $N \geq 2$, let $\operatorname{PVect}(X, D, N) \subseteq \operatorname{PVect}(X, D)$ denote the subcategory consisting of all parabolic vector bundles all of whose parabolic weights are multiples of $1 / N$. It is straight-forward to check that $\operatorname{PVect}(X, D, N)$ is closed under all the above operations, namely parabolic tensor product, direct sum and taking the parabolic dual.

28 (The covering construction). Let $X$ be a smooth projective variety and $D$ an effective simple normal crossing divisor. The Covering Lemma of Kawamata [Kaw81, Thm.17] says that there is a connected smooth projective variety $Y$ and a Galois covering morphism

$$
p: Y \quad \longrightarrow \quad X
$$

such that the reduced divisor $\tilde{D}:=\left(p^{*} D\right)_{\text {red }}$ is a normal crossing divisor on $Y$ and furthermore, $p^{*} D_{i}=k_{i} N \cdot\left(p^{*} D_{i}\right)_{r e d}$, where the $k_{i}$ are positive integers. Let $G$ denote the Galois group for the covering map $p$.

Definition 29 (The category of $G$-bundles). $\quad$ Let $G \subseteq \operatorname{Aut}(Y)$ be a finite subgroup of the group of automorphisms of a connected smooth projective variety $Y$. The natural action of $G$ on $Y$ is encoded in a morphism

$$
\mu: G \times Y \longrightarrow Y .
$$

Let $\operatorname{Vect}_{G}(Y)$ denote the category of all $G$-linearized vector bundles on $Y$. The isotropy group of any point $y \in Y$, for the action of $G$ on $Y$, will be denoted by $G_{y}$.

Let $\operatorname{Vect}_{G}^{D}(Y, N)$ denote the subcategory of $\operatorname{Vect}_{G}(Y)$ consisting of all $G$ linearized vector bundles $W$ over $Y$ satisfying the following three conditions:

(1) for a general point $y$ of an irreducible component of $\left(p^{*} D_{i}\right)_{\text {red }}$, the isotropy subgroup $G_{y}$ is cyclic of order $\left|G_{y}\right|=n_{y}$ which is a divisor of $N$; the action of the isotropy group $G_{y}$ on the fiber $W_{y}$ is of order $N$, which is equivalent to the condition that for any $g \in G_{y}$, the action of $g^{N}$ on $W_{y}$ is the trivial action;

(2) The action is given by a representation $\rho_{y}$ of $G_{y}$ given by a block diagonal matrix

$$
\rho_{y}(\zeta)=\operatorname{diag}\left(z^{d_{1}} I_{1}, \ldots, z^{d_{l}} I_{l}\right)
$$

where $\zeta$ is a generator of the group $G_{y}$ and whose order $n_{y}$ divides $N$ and $0 \leq d_{1}<d_{2}<\cdots<d_{l} \leq n_{y}-1, I_{j}$ is the identity matrix of order $r_{j}$ and $z$ is an $n_{y}$-th root of unity. 
If $N=s \cdot n_{y}$ and $m_{j}=s \cdot d_{j}$ for $j=1, \ldots l$, then $\alpha_{i}=\frac{d_{i}}{n_{y}}=\frac{m_{i}}{N}$ and $r_{j}$ is the multiplicity of the weight $\alpha_{j}$. Note that $0 \leq m_{1}<m_{2}<$ $\ldots<m_{l} \leq N-1$.

(3) For a general point $y$ of an irreducible component of a ramification divisor for $p$ not contained in $\left(p^{*} D\right)_{\text {red }}$, the action of $G_{y}$ on $W_{y}$ is the trivial action.

Following Seshadri [Ses70, p.161] we call the $G$-bundles $E$ in $\operatorname{Vect}_{G}^{D}(Y, N)$ bundles of fixed local orbifold type $\tau$.

We remark that this definition of $G$-bundles of fixed local type easily extends to $G$-torsion-free sheaves since the local action is specified only at the generic points of the parabolic divisor.

We note that $\operatorname{Vect}_{G}^{D}(Y, N)$ is also an additive tensor category.

30 (Parabolic bundles and $G$-bundles). In [Bis97] an identification between the objects of $\operatorname{PVect}(X, D, N)$ and the objects of $\operatorname{Vect}_{G}^{D}(Y, N)$ has been constructed. Given a $G$-homomorphism between two $G$-linearized vector bundles, there is a naturally associated homomorphisms between the corresponding vector bundles, and this identifies, in a bijective fashion, the space of all $G$-homomorphisms between two objects of $\operatorname{Vect}_{G}^{D}(Y, N)$ and the space of all homomorphisms between the corresponding objects of $\operatorname{PVect}(X, D, N)$. An equivalence between the two additive tensor categories, namely $\operatorname{PVect}(X, D, N)$ and $\operatorname{Vect}_{G}^{D}(Y, N)$, is obtained this way.

We observe that an earlier assertion that the parabolic tensor product operation enjoys all the abstract properties of the usual tensor product operation of vector bundles, is a consequence of the fact that the above equivalence of categories indeed preserves the tensor product operation.

The above equivalence of categories has the further property that it takes the parabolic dual of a parabolic vector bundle to the usual dual of the corresponding $G$-linearized vector bundle.

Definition 31 (Stable parabolic bundles). The definition of parabolic semistable and parabolic polystable vector bundles is given in MaruyamaYokogawa [MY92] and Mehta-Seshadri [MS80]. Given an ample divisor $H$, the parabolic degree of a parabolic bundle $E_{*}$ is defined by

$$
\operatorname{pardeg}\left(E_{*}\right):=\int_{0}^{1} \operatorname{deg}\left(E_{t}\right) d t+r \cdot \operatorname{deg}(D)
$$


where $E_{t}$ comes from the filtration defined in (26.1). There is a natural notion of parabolic subsheaf and given any subsheaf of $E$ there is a canonical parabolic structure that can be given to this subsheaf. (cf [MY92], [Bis97] for details)

A parabolic bundle is called stable (resp. semistable) if for any proper nonzero coherent parabolic subsheaf $V_{*}$ of $E_{*}$ with $0<\operatorname{rank}\left(V_{*}\right)<\operatorname{rank}\left(E_{*}\right)$, with $E / V$ being torsion free, the following inequality is valid:

$$
\frac{\operatorname{pardeg} V}{\operatorname{rank} V}<\frac{\operatorname{pardeg} E}{\operatorname{rank} E} \quad \operatorname{resp.} \frac{\operatorname{pardeg} V}{\operatorname{rank} V} \leq \frac{\operatorname{pardeg} E}{\operatorname{rank} E} .
$$

Remark 32. If we work with the definition given in (27), then we have the following expression for parabolic degree of $E_{*}$ which is along the lines of [MS80]. Define:

$$
\operatorname{weight}\left(E_{*}\right):=\sum_{i, j} \alpha_{j}^{i}\left(c_{1}\left(\mathcal{F}_{i}^{j}(E)\right) \cdot H^{n-1}-c_{1}\left(\mathcal{F}_{i}^{j+1}(E)\right) \cdot H^{n-1}\right) .
$$

Using the fact that on the divisor $D, c_{1}(F)=r k(F) D$, we have the following expression:

$$
\operatorname{weight}\left(E_{*}\right):=\sum_{i, j} \alpha_{j}^{i}\left[\operatorname{rank}\left(\mathcal{F}_{i}{ }^{j}(E)\right)-\operatorname{rank}\left(\mathcal{F}_{i}{ }^{j+1}(E)\right)\right]\left(D_{i} \cdot H^{n-1}\right) .
$$

Then it is not hard to check that the parabolic degree of $E_{*}$ is given by:

$$
\operatorname{pardeg}\left(E_{*}\right)=\operatorname{deg}(E)+\operatorname{weight}\left(E_{*}\right) \text {. }
$$

Definition 33 (Stable $G$-bundles). A $G$-linearized vector bundle $V^{\prime}$ over $Y$ is called $(G, \mu)$-stable (resp. $(G, \mu)$-semistable) if for any proper nonzero coherent subsheaf $F^{\prime} \subset V^{\prime}$, invariant under the action of $G$ and with $V^{\prime} / F^{\prime}$ being torsion free, the following inequality is valid:

$$
\frac{\operatorname{deg} F^{\prime}}{\operatorname{rank} F^{\prime}}<\frac{\operatorname{deg} V^{\prime}}{\operatorname{rank} V^{\prime}} \quad \operatorname{resp} . \quad \frac{\operatorname{deg} F^{\prime}}{\operatorname{rank} F^{\prime}} \leq \frac{\operatorname{deg} V^{\prime}}{\operatorname{rank} V^{\prime}}
$$

The $G$-linearized vector bundle $V^{\prime}$ is called $G$-polystable if it is a direct sum of $G$-stable vector bundles of same slope, where, as usual, slope $:=$ degree/rank.

Remark 34. $G$-invariant subsheaves of $V^{\prime}$ are in one-to-one correspondence with the subsheaves of the parabolic vector bundle corresponding to $V^{\prime}$, and furthermore, the degree of a $G$-invariant subsheaf is simply the order of $G$-times the parabolic degree of the corresponding subsheaf with the induced parabolic structure [Bis97].

It is immediate that $V^{*}$ is $G$-semistable if and only if $V$ is so. 
The above equivalence of categories between $\operatorname{PVect}(X, D, N)$ and $\operatorname{Vect}_{G}^{D}(Y$, $N$ ) in fact identifies the subcategory of parabolic stable bundles with the $G$ stable bundles. This result, due to Biswas, generalizes the result of Seshadri for parabolic bundles over curves (cf. [Bis97], [Ses70]).

Proposition 35. Let $E$ be a stable vector bundle on $X$ with $\operatorname{rank}(E)=$ $n$ and $\operatorname{deg}(E)=q$ and such that $-n<q \leq 0$. Then, for any smooth divisor $D \subset X$ such that $D \in|H|$, one can endow $E$ with a parabolic structure along $D$ such that $\operatorname{pardeg}(E)=0$ and $E$ is parabolic stable with this structure.

Proof. Let $p: Y \rightarrow X$ be a Kawamata cover of $X$ with Galois group $G$ and ramification index along $D$ to be the integer $n$. Define $\tilde{D}:=\left(p^{*}(D)\right)_{\text {red }}$ so that $p^{*}(D)=n \cdot\left(p^{*}(D)\right)_{\text {red }}$. Further, in the notation of (29), the weight $\alpha$ attached to the action of the isotropy $G_{y}$ at a general point $y \in \tilde{D}$ is given by $\alpha=\frac{-q}{n}$.

Since $\tilde{D}$ is invariant under the action of $G$, for any $k \in \mathbf{Z}$, the line bundle $\mathcal{O}_{Y}(k \tilde{D})$ gets a structure of a $G$-bundle.

Define $\mathbf{L}=\mathcal{O}_{Y}(-q \cdot \tilde{D})$. Then $\mathbf{L}$ also gets a $G$-bundle structure. Now consider the $G$-bundle $p^{*}(E)$ and let $W$ be the $G$-bundle (of type $\tau$ in the notation of (29) defined by:

$$
W=p^{*}(E) \otimes_{\mathcal{O}_{Y}} \mathbf{L} .
$$

It is easy to see that $p_{*}^{G}(W)=E$. Further, $E$ realised as the invariant direct image of $W$ gets a natural parabolic structure, called the special parabolic structure where the flag has only two terms

$$
\left.E\right|_{D}=\mathcal{F}_{D}^{1}(E) \supset \mathcal{F}_{D}^{2}(E)=0
$$

with weight $\alpha=\frac{-q}{n}$.

The parabolic degree of $E$ with this structure is given by:

$$
\operatorname{pardeg}(E)=\operatorname{deg}(E)+n \cdot \alpha=\operatorname{deg}(E)-q=0 .
$$

We observe that for any subbundle $V \subset E$ with $\operatorname{rank}(V)=r$, there is a unique way of defining the induced special parabolic structure on $V$ and pardeg $(V)=$ $\operatorname{deg}(V)+r \cdot \alpha=\operatorname{deg}(V)+r \cdot \frac{-q}{n}$. Hence,

$$
\frac{\operatorname{pardeg}(V)}{r}=\frac{\operatorname{deg}(V)}{r}+\frac{-q}{n}<\frac{\operatorname{pardeg}(E)}{n}=0
$$

since $E$ is stable. Thus, we conclude that $E$ is parabolic stable with this parabolic structure. We also note that by the correspondence between parabolic 
stable bundles on $X$ and $(G, \mu)$-stable bundles on $Y$ (Remark 34), the $G$-bundle $W$ is $(G, \mu)$-stable.

Remark 36. This proposition can be seen in the more general context of parabolic bundles. Let $E_{*} \in \operatorname{Vect}(X, D)$ with $\operatorname{pardeg}\left(E_{*}\right) \neq 0$. Then there exists a parabolic bundle $E_{*}^{\prime} \in \operatorname{Vect}\left(X, D^{\prime}\right)$, where $D \subset D^{\prime}$ and $D^{\prime}$ has more components $\Delta_{j}$ meeting $D_{i}$ with simple normal crossing singularities, such that

$$
\operatorname{pardeg}\left(E_{*}^{\prime}\right)=0
$$

and further, the forgetful functor

$$
V e c t\left(X, D^{\prime}\right) \rightarrow \operatorname{Vect}(X, D), \quad E_{*}^{\prime} \rightarrow E_{*}
$$

is fully faithful and preserves parabolic semistability and parabolic stability.

To see this, we define $E_{*}^{\prime}=\left(E^{\prime}, F_{*}^{\prime}, \alpha_{*}^{\prime}\right)$ as follows:

Assume that $\operatorname{pardeg}\left(E_{*}\right)<0$. This is always possible to achieve by twisting with a line bundle. Let integers $m_{j}>0$ be so chosen, such that for rational numbers $0 \leq \beta_{j}<1$, we have the equality

$$
\left(\sum\left(m_{j} \beta_{j}\left(\Delta_{j} \cdot H^{n-1}\right)\right)=\frac{-\operatorname{pardeg}\left(E_{*}\right)}{\operatorname{rank}(E)} .\right.
$$

Let $D^{\prime}=\sum_{i=1}^{n} D_{i}+\sum_{j=1}^{m} \Delta_{j}$ and $E^{\prime}=E$. Define the filtration as follows:

$$
\left.E^{\prime}\right|_{\Delta_{j}}=\mathcal{F}_{0}{ }^{1}\left(E^{\prime}\right) \supset \mathcal{F}_{0}{ }^{2}\left(E^{\prime}\right)=(0)
$$

with a single weight $\beta_{j}$ for each $1 \leq j \leq m$. That is, $\left(\alpha_{*}^{\prime}\right)=\left(\alpha_{*}\right) \cup\left(\beta_{*}\right)$. Clearly, weight $\left(E_{*}^{\prime}\right)=\operatorname{weight}\left(E_{*}\right)+\operatorname{rank}(E)\left[\sum\left(m_{j} \beta_{j}\left(\Delta_{j} \cdot H^{n-1}\right)\right]\right.$. Hence, $\operatorname{pardeg}\left(E_{*}^{\prime}\right)=\operatorname{pardeg}\left(E_{*}\right)+\operatorname{rank}(E)\left[\sum\left(m_{j} \beta_{j}\left(\Delta_{j} \cdot H^{n-1}\right)\right]=0\right.$.

The above Proposition is used to extend our theory of holonomy to parabolic stable bundles. Before we do that, we need to prove the MehtaRamanathan restriction theorem for $G$-torsion free sheaves. By the equivalence of categories between $G$-bundles and parabolic bundles, we get a MehtaRamanathan-type restriction theorem for parabolic bundles.

Theorem 37 (The $G$-Mehta-Ramanathan theorem). Let $E$ be a $(G$, $\mu$ )-semistable (resp. stable) $G$-torsion free sheaf on a normal projective $G$ variety. Then the restriction $\left.E\right|_{C}$ to a general complete intersection $G$-curve $C$ of large degree (with respect to the pull-back line bundle $p^{*}(H)$ ) is $(G, \mu)$ semistable (resp. stable). 
Proof. Since $(G, \mu)$-semistability for $G$-sheaves is equivalent to the semistability of the underlying sheaf, the non-trivial case is that of stability. The proof follows from the following observations:

(1) $E$ is $(G, \mu)$-stable iff $E$ is polystable and $\operatorname{Hom}(E, E)^{G}$ is 1-dimensional.

Indeed, we noted that $E$ is semistable. If $E$ is not polystable then it has a nontrivial socle $F \subset E$ with $\mu(F)=\mu(E)$ which is invariant under all the automorphisms of $E$, in particular invariant under the group $G$ (cf. [HL97, 1.5.5]). This contradicts the $G$ stability of $E$.

(2) By the orbifold version of the Enriques-Severi theorem, for sufficiently high degree complete intersection $G$-curve $C, \operatorname{Hom}_{X}(E, E)=$ $\operatorname{Hom}_{C}\left(\left.E\right|_{C},\left.E\right|_{C}\right)$ and so $\operatorname{Hom}_{X}(E, E)^{G}=\operatorname{Hom}_{C}\left(\left.E\right|_{C},\left.E\right|_{C}\right)^{G}$.

(3) Finally, by the restriction theorem of Bogomolov (cf. [HL97, Sec.7.3]), for every complete intersection curve $C$ in the linear system $|m H|$ (the number $m$ being effectively determined), the restriction $\left.E\right|_{C}$ is polystable. Thus we can work with general high degree complete intersection $G$-curves rather than with general complete intersection curves.

We can now define the holonomy groups of parabolic stable bundles.

Definition 38 (Holonomy groups of parabolic stable bundles). Let $E$ be a $(G, \mu)$-stable bundle on $Y$ of degree 0 . This corresponds to a parabolic bundle on $X$ of parabolic degree 0 . Let $C_{k}$ be a general CI curve in $Y$ which is $G$-invariant.

The quotient $C_{k} / G=: T_{k}$ is also a smooth projective curve in $X$. By choosing $C_{k}$ sufficiently general, one can make sure that the action of $G$ on $C_{k}$ is faithful and we can realize the group $G$ as a quotient $\Gamma / \Gamma_{o}$, where $\Gamma_{o}=\pi_{1}\left(C_{k}\right)$ and $\Gamma$ acts properly discontinuously on the simply connected cover $\tilde{C}_{k}$ and $T_{k}=\tilde{C}_{k} / \Gamma$. (The $\Gamma$-action on $\tilde{C}_{k}$ is not assumed to be free.)

By the restriction theorem above, $\left.E\right|_{C_{k}}$ is a $(G, \mu)$-stable bundle on $C_{k}$, hence it comes from an irreducible unitary representation of the group $\rho: \Gamma \rightarrow$ $G L\left(E_{y}\right)$, for $y \in C_{k} \subset Y$ a point away from the ramification locus. We note that an irreducible unitary representation of $\Gamma$ descends to a bundle on $\tilde{C}_{k} / \Gamma_{o}=C_{k}$ which comes with a $G$-action.

Now by considering the map $p: C_{k} \rightarrow T_{k}$ and taking the invariant direct image $p_{*}^{G}\left(\left.E\right|_{C_{k}}\right)$ we get a bundle $F$ which is parabolic stable on $T_{k}$, with parabolic structure on $T_{k} \cap D$. Hence, as above, the group $\Gamma$ which acts on the 
simply connected curve $\tilde{C}_{k}$ with parabolic fixed points such that $p_{*}^{G}\left(\left.E\right|_{C_{k}}\right)=F$ arises from a unitary representation $\rho: \Gamma \rightarrow G L\left(F_{x}\right)$, with $p(y)=x$.

Let $y \in C_{k} \subset Y$ be a point away from the ramification locus. The arguments in Section 2 now imply that the Zariski closure of $\operatorname{im}(\rho)=H_{y}$ is well-defined and is the smallest reductive subgroup of $G L\left(E_{y}\right)$ such that the $G$-bundle $E$ has a reduction of structure group to $H_{y}$ and the reduction is moreover $G$-invariant. Moreover, $H_{y}$ can be identified with the Zariski closure of the image of $\rho$ in $G L\left(F_{x}\right)$ where $p(y)=x$.

By the categorical equivalence between $G$-bundles on $Y$ and parabolic bundles on $X$, it follows that the group $H_{y}=H_{x}$ is realized as the holonomy group of the parabolic bundle $p_{*}^{G}(E)$ on $X$. This defines the holonomy group for all parabolic stable bundles in the category $\operatorname{PVect}(X, D)$.

Remark 39. If the bundle arises as an irreducible representation of $\pi_{1}(X \backslash D)$ then the resulting parabolic bundle will have all parabolic Chern classes zero and this fits into the theme addressed by Deligne in [Del70].

\section{§4. Computing the Holonomy Group}

Given a stable vector bundle $E$, the computation of its holonomy group seems quite hard in general. The definition (19) is practically impossible to use. The method of Tannaka duality [Tan38] shows that one can determine the holonomy once we know the decomposition of $E^{\otimes m} \otimes\left(E^{*}\right)^{\otimes n}$ into direct summands for every $m, n$. The observation of Larsen (which seems to be unpublished) is that one can frequently characterize a subgroup $G \subset G L(V)$ by knowing the decomposition of the $G$-module $V^{\otimes m} \otimes\left(V^{*}\right)^{\otimes n}$ for only a very few values of $m, n$.

Our aim is to translate this into geometric form and give several examples illustrating the principle (3). Let us start with the general form of (4).

Lemma 40. Notation and assumptions as in (19). There is an étale cover $\pi: U \rightarrow(X \backslash \operatorname{Sing} X)$ with Galois group $H_{x}(E) / H_{x}(E)^{0}$ such that the holonomy group of $\pi^{*} E$ is $H_{x}(E)^{0}$, hence connected.

Proof. Let $B \subset X$ be the set of points where either $X$ is singular or $E$ is not locally free. To the vector bundle $\left.E\right|_{X \backslash B}$ we can associate a principal $H_{x}(E)$-bundle $P \rightarrow(X \backslash B)$. Then $U:=P / H_{x}(E)^{0} \rightarrow(X \backslash B)$ is an étale cover with Galois group $H_{x}(E) / H_{x}(E)^{0}$. Since $\pi^{*} P / H_{x}(E)^{0} \rightarrow U$ has a section, the structure group of $\pi^{*} P$ can be further reduced to $H_{x}(E)^{0}$.

The following result relates the holonomy groups to symmetric powers. 
Proposition 41. Notation as in (19). Assume that $X \backslash B$ is simply connected. Then the following are equivalent:

(1) The reflexive hull of $S^{m} E$ is indecomposable for some $m \geq 2$.

(2) The reflexive hull of $S^{m} E$ is indecomposable for every $m \geq 2$.

(3) The holonomy is one of the following:

(a) $S L\left(E_{x}\right)$ or $G L\left(E_{x}\right)$,

(b) $S p\left(E_{x}\right)$ or $G S p\left(E_{x}\right)$ for a suitable nondegenerate symplectic form on $E_{x}$ (and $\operatorname{rank} E$ is even).

Proof. Let $\mathfrak{h} \subset \mathfrak{g} l\left(E_{x}\right)$ denote the Lie algebra of $H_{x}(E)$. A representation of $H_{x}(E)$ is indecomposable iff the corresponding representation of $\mathfrak{h}$ is indecomposable. Thus (41) is equivalent to the corresponding statement about Lie algebras. The latter is a special case of [Sei87, §17,Thm.1]

The following is a key example in relating the holonomy groups to geometric structures.

Example 42. Let $E$ be a rank 3 bundle with holonomy group $S_{3}$ over a smooth projective variety $X$.

We can also think of the standard representation of $S O_{3} \cong P S L_{2}$ as the symmetric square of the standard representation of $S L_{2}$. Does this mean that every rank 3 bundle with $\mathrm{SO}_{3}$-holonomy can be written as the symmetric square of a rank 2 bundle with $S L_{2}$-holonomy?

Principal $P S L_{2}$-bundles are classified by $H_{e t}^{1}\left(X, P S L_{2}\right)$. The obstruction to lift to a principal $S L_{2}$-bundle is in $H_{e t}^{2}\left(X, \mu_{2}\right)$, which is never zero. (For a basic reference, see [Mil80, Sec.IV.2].)

To put it in more concrete terms, observe that for any rank 2 bundle $F$, the rank 3 bundle $S^{2} \mathrm{~F} \otimes \operatorname{det}^{-1} \mathrm{~F}$ has trivial determinant and $\mathrm{SO}_{3}$-holonomy. If $\operatorname{det} F \cong L^{2}$ is the square of a line bundle, then

$$
S^{2} F \otimes \operatorname{det}^{-1} F \cong S^{2}\left(F \otimes L^{-1}\right),
$$

but if $\operatorname{det} F$ is not the square of a line bundle, then there does not seem to be any natural way to write $S^{2} F \otimes \operatorname{det}^{-1} F$ as a symmetric square.

This is the obstruction in $\operatorname{Pic}(X) / \operatorname{Pic}(X)^{2} \subset H_{e t}^{2}\left(X, \mu_{2}\right)$ that we detected earlier.

This suggests that it is easier to lift a $P S L_{2}$-bundle to a $G L_{2}$-bundle tensored with a line bundle than to an $S L_{2}$-bundle. This turns out to be a general pattern, which we study next. 
43 (Holonomy groups and representations of classical groups). Here we study vector bundles $E$ over a projective variety $X$ whose holonomy group is contained in an irreducible representation of a product

$$
\rho: G=G_{0} \times \prod_{i=1}^{m} G_{i} \rightarrow H \supset H_{x}(E) .
$$

Where $G_{0}$ is a subgroups of the scalars in $G L\left(E_{x}\right)$ and for $i \geq 1, G_{i}$ is one of the classical groups $S L_{n_{i}}, S p_{n_{i}}, S O_{n_{i}}$. Thus $\rho$ can be obtained from the basic representations of the $G_{i}$ by a tensor product of Schur functors $\mathbb{S}_{i}$.

The easy case is when $\rho$ is an isomorphism. In this case $E$ corresponds to a principal $G$-bundle and the basic representation of each $G_{i}$ gives a vector bundle $F_{i}$ of rank $n_{i}$ with structure group $G_{i}$. Here $L \cong F_{0}$ is a line bundle. Thus we obtain that

$$
E \cong L \otimes \bigotimes_{i \geq 1} \mathbb{S}_{i}\left(F_{i}\right)
$$

The situation is more complicated if $\operatorname{ker} \rho \neq 1$. To $E$ we can associate a principal $H$-bundle and the obstruction to lift it to a principal $G$-bundle lies in $H_{e t}^{2}(X, \operatorname{ker} \rho)$. If $\operatorname{ker} \rho$ is not connected, then this is never zero.

We can improve the situation by replacing the groups

$$
S L_{n_{i}}, S p_{n_{i}}, S O_{n_{i}} \quad \text { by } \quad G L_{n_{i}}, G S p_{n_{i}}, G S O_{n_{i}}
$$

and extending $G_{0}$ to all scalars $\mathbb{C}^{*}$. Let us denote these groups by $G_{i}^{*}$. Set $G^{*}:=\prod_{i \geq 0} G_{i}^{*}$ and extend $\rho$ to $\rho^{*}=G^{*} \rightarrow \mathbb{C}^{*} \cdot H$.

Since $G_{0}$ maps isomorphically onto the scalars, we see that

$$
\operatorname{ker} \rho^{*} \cong \prod_{i \geq 1} Z\left(G_{i}^{*}\right) \cong\left(\mathbb{C}^{*}\right)^{m}
$$

The obstruction to lift a principal $\mathbb{C}^{*} \cdot H$-bundle to a principal $G^{*}$-bundle is now in the Brauer group $\operatorname{Br}(X):=H_{\text {et }}^{2}\left(X, \mathcal{O}_{X}^{*}\right)$.

Therefore, if the Brauer group is zero, then we can lift our principal $H$ bundle to a principal $G^{*}$-bundle. Thus, as before, we obtain the following:

Proposition 44. Let $X$ be a smooth projective variety such that $H^{2}(X$, $\left.\mathcal{O}_{X}\right)=0$ and $H^{3}(X, \mathbb{Z})$ is torsion free.

Let $E$ be a stable vector bundle of rank $N$ on $X$ whose holonomy group is contained in the image of an irreducible representation

$$
\rho: G=\prod_{i=1}^{m} G_{i} \rightarrow G L_{N}
$$


given by Schur functors $\mathbb{S}_{i}$, where each $G_{i}$ is one of the groups $G L_{n_{i}}, G S p_{n_{i}}$, $G S O_{n_{i}}$. Then there are vector bundles $F_{i}$ of rank $n_{i}$ with structure group $G_{i}$, and a line bundle $L$ such that

$$
E \cong L \otimes \bigotimes_{i} \mathbb{S}_{i}\left(F_{i}\right)
$$

Next we illustrate the principle (3) by studying the possible holonomy groups and the corresponding geometric structures for bundles of small rank.

45 (Holonomy groups of low rank bundles). Let $X$ be a smooth projective variety and $E$ a vector bundle which is stable with respect to an ample divisor $H$. Assume that the holonomy group $H_{x}(E)$ is connected.

Rank 2 bundles. Here $H_{x}(E)$ is $S L_{2}$ or $G L_{2}$. The first case corresponds to $\operatorname{det} E \cong \mathcal{O}_{X}$ and the second to the case when $\operatorname{det} E$ is a line bundle which is not torsion in $\operatorname{Pic} X$.

The case when $\operatorname{det} E$ is torsion in $\operatorname{Pic} X$ would give nonconnected holonomy.

Rank 3 bundles. The general case is when $H_{x}(E)$ is $S L_{3}$ or $G L_{3}$. We can also have $S_{3}$, when $E \cong E^{*}$ or $G S O_{3}$ when $E \cong E^{*} \otimes L$ for some line bundle $L$.

The isomorphism $S O_{3} \cong P S L_{2}$ was studied in (42).

Rank 4 bundles. The cases when $H_{x}(E)$ is $S L_{4}, G L_{4}$ or $S O_{4}, G S O_{4}$ are as before. We can also have $S p_{4}$ or $G S p_{4}$ holonomy, corresponding to the existence of a skew symmetric pairing $E \times E^{*} \rightarrow \mathcal{O}_{X}$ or $E \times E^{*} \rightarrow L$ for some line bundle $L$.

There are 2 more interesting cases when $H_{x}(E)=S L_{2}$ or $H_{x}(E)=$ $G L_{2} / \mu_{3}$ with the 3 rd symmetric power representation.

Assume that $H_{x}(E)=S L_{2}$ with the 3 rd symmetric power representation. Then $H_{x}(E) \subset S p_{4}$. Furthermore, by (43), there is a rank 2 vector bundle $F$ such that $E \cong S^{3} F$.

Finally the last case is when $H_{x}(E)=G L_{2} / \mu_{3}$. This can be treated as in (44).

Rank 5 bundles. The cases when $H_{x}(E)$ is $S L_{5}, G L_{5}$ or $S O_{5}, G S O_{5}$ are as before.

There are 2 other cases when $H_{x}(E)=P S L_{2}$ or $H_{x}(E)=G L_{2} / \mu_{4}$ with the 4 th symmetric power representation. We see that $P S L_{2} \subset S O_{5}$ and and $G L_{2} / \mu_{4} \subset G S O_{5}$, so these have orthogonal structures. A more detailed study is given in (44). 
Rank 6 bundles. The cases when $H_{x}(E)$ is $S L_{6}, G L_{6}, S O_{6}, G S O_{6}$ or $S p_{6}$, $G S p_{6}$ are as before.

The cases when $H_{x}(E)=S L_{2}$ or $H_{x}(E)=G L_{2} / \mu_{5}$ with the 5 th symmetric power representation or $H_{x}(E)=S L_{3}$ or $H_{x}(E)=G L_{3} / \mu_{2}$ with the 2 nd symmetric power representation work as the rank 4 cases.

By luck, the cases when $H_{x}(E)=S L_{4} / \mu_{2}$ or $H_{x}(E)=G L_{4} / \mu_{2}$ with the 2nd exterior power representation are contained in $S_{6}$ (resp. $\left.G S O_{6}\right)$.

The last case is when $H_{x}(E)=S L_{2} \times S L_{3}$, or $H_{x}(E)=G L_{2} \times G L_{3} / \mathbb{C}^{*}$ with the tensor product of the standard representations. In the first case, by (43), we get that $E \cong F_{2} \otimes F_{3}$ with $H_{x}\left(F_{2}\right)=S L_{2}$ and $H_{x}\left(F_{3}\right)=S L_{3}$ while in the second case we again have a Brauer obstruction to deal with (44).

Every other connected reductive subgroup of $G L_{6}$ is contained in one of the above.

Rank 7 bundles. The cases when $H_{x}(E)$ is $S L_{7}, G L_{7}$ or $S O_{7}, G S O_{7}$ are as before.

The cases when $H_{x}(E)=P S L_{2}$ or $H_{x}(E)=G L_{2} / \mu_{6}$ with the 6 rd symmetric power representation are examined in (44).

The first exceptional case also appears, namely we can have monodromy group $G_{2} \subset S O_{7}$ or $\mathbb{C}^{*} \cdot G_{2} \subset G S O_{7}$. We can not say anything useful about it beside noting that the monodromy is a subgroup of $\mathbb{C}^{*} \cdot G_{2}$ iff $\wedge^{3} E$ has a line bundle direct summand. This is a consequence of the corresponding characterization of $G_{2} \subset S L_{7}$ as the subgroup that fixes a general skew symmetric trilinear form.

Indeed, one checks that in the $S L_{7}, G_{7}, S O_{7}, G S O_{7}$ cases there is no 1dimensional invariant subspace in $\wedge^{3} E$. In the $P S L_{2}$ or $G L_{2} / \mu_{6}$ cases there is such an invariant subspace, but $P S L_{2} \subset G_{2}$ and $G L_{2} / \mu_{6} \subset \mathbb{C}^{*} \cdot G_{2}$.

Rank 8 bundles. Here we get the first case of a bundle $E$ with $c_{1}(E)=0$ where the pattern of (7) no longer holds.

This is when the holonomy group is the tensor product of the standard representations of $S L_{2}$ and $S L_{4}$. Thus $H_{x}(E) \cong\left(S L_{2} \times S L_{4}\right) / \mu_{2}$ and the Brauer obstruction is inevitable.

Rank $\leq 16$ bundles. By now it should be clear that one can continue in this manner for low ranks, and either direct constructions or the method of (44) apply.

For rank 16 we run into the first case where the holonomy can be a spinor group, here $H_{x}(E) \cong \operatorname{Spin}_{5}$. Probably it is again a Brauer-type obstruction, whose vanishing ensures that $E$ is one of the half spin subbundles of the Clifford algebra of a rank 5 bundle $F$ with orthogonal structure. 


\section{$\S 5 . \quad$ Tangent Bundles}

It may be especially interesting to consider the holonomy group of the tangent bundle $T_{X}$ of a smooth projective variety $X$. There are only a few cases when we can compute the algebraic holonomy group.

46 (Calabi-Yau varieties). Let $X$ be a simply connected smooth projective variety $X$ such that $K_{X}=0$ which is not a direct product.

The differential geometric holonomy group is either $S U_{n}(\mathbb{C})$ or $U_{n}(\mathbb{H})$. As observed in [Bea83], the tensor powers of $T_{X}$ decompose according to the representation theory of $S U_{n}$ (resp. or $U_{n}(\mathbb{H})$ ), thus by (2.3) we conclude that the algebraic holonomy is $S L_{n}(\mathbb{C})\left(\right.$ resp. $S p_{2 n}(\mathbb{C})$ ). This proves $(10)$.

47 (Homogeneous spaces). Let $X=G / P$ be a smooth, projective homogeneous space under a reductive group $G$. Let $\rho: P \rightarrow G L\left(T_{x} X\right)$ denote the stabilizer representation. The stabilizer representation vanishes on the unipotent radical $U \subset P$ and so we can view $\rho$ as a representation of the reductive Levi subgroup $\rho: P / U \rightarrow G L\left(T_{x} X\right)$.

The tangent bundle $T_{X}$ is indecomposable iff $\rho$ is irreducible. By [Ram66], [Ume78], [Kob86], in this case $T_{X}$ is stable and tensor powers of $T_{X}$ decompose according to the representation theory of $P / U$. Thus by (2.3) we conclude that the algebraic holonomy group is $\rho(P / U)$. This proves (11).

There are very few examples of Fano varieties whose holonomy group we can compute.

For instance, let $S$ be a Del Pezzo surface which is obtained from $\mathbb{P}^{2}$ by blowing up at least 3 points. It is easy to see that $T_{S}$ is stable, hence by (4) the holonomy group is $G L_{2}$.

Question 48. Let $X^{n}$ be a smooth projective variety with Picard number 1 and $-K_{X}$ ample. Assume that the automorphism group of $X$ is finite and $T_{X}$ is stable. Is the algebraic holonomy group $G L_{n}$ ?

49 (Varieties with ample canonical class). Let $X$ be a smooth projective variety $X$ such that $K_{X}$ is ample.

By the Akizuki-Nakano vanishing theorem (cf. [GH94, p.155]), $\wedge^{i} \Omega_{X}$ contains no ample line bundle for $i<\operatorname{dim} X$. In particular, $\Omega_{X}$ does not contain any subsheaf of rank $<\operatorname{dim} X$ whose determinant is ample and so it is stable with respect to the ample divisor $K_{X}$. (This also follows from the much stronger result of Aubin and Yau about the existence of a Kähler-Einstein metric.)

Furthermore, this also implies that $\wedge^{i} \Omega_{X}$ has no line bundle direct summands for $i<\operatorname{dim} X$. Thus we conclude: 
Proposition 50. Let $X$ be a smooth projective variety $X$ such that $K_{X}$ is ample and let $H_{x} \subset G L\left(T_{x} X\right)$ denote the holonomy group of the tangent bundle $T_{X}$.

Then $\wedge^{i} T_{x} X$ has no 1-dimensional $H_{x}$-invariant subspaces for $i<\operatorname{dim} X$. In particular, $H_{x}$ acts irreducibly on $T_{x} X$.

Thus it is natural to study subgroups $H \subset G L(E)$ such that $\wedge^{i} E$ has no 1-dimensional $H$-invariant subspace for $i<\operatorname{dim} E$. This is a very restrictive condition, but we have not been able to classify all such representations. In any case, at the moment we do not even know the answer to the following:

Question 51. Is there a simply connected, smooth projective variety $X$ with Picard number 1 and $K_{X}$ ample, whose algebraic holonomy group is different from $G L_{n}$ ?

There are smooth projective varieties with Picard number 1 which are quotients of a direct product, and these have smaller holonomy group.

\section{Acknowledgments}

We thank F. Bogomolov, R. Guralnick, R. Lazarsfeld, R. Livné, P. Sarnak and C.S Seshadri for useful comments, references and suggestions. We are grateful for many discussions with M. Larsen concerning representation theory. Partial financial support for JK was provided by the NSF under grant number DMS-0500198.

\section{References}

[Bal05] V. Balaji, Principal bundles on projective varieties and the Donaldson-Uhlenbeck compactification, J. Differential Geom. 76 (2007), no. 3, 351-398.

[Bea83] A. Beauville, Variétés Kähleriennes dont la première classe de Chern est nulle, J. Differential Geom. 18 (1983), no. 4, 755-782 (1984).

[Bis97] I. Biswas, Parabolic bundles as orbifold bundles, Duke Math. J. 88 (1997), no. 2, 305-325.

[Bis07] , On the algebraic holonomy of stable principal bundles. arXiv:math. AG/0703104, 2007.

[Bog94] F. A. Bogomolov, Stable vector bundles on projective surfaces, Mat. Sb. 185 (1994), no. 4, 3-26; translation in Russian Acad. Sci. Sb. Math. 81 (1995), no. 2, 397-419.

[Bry00] R. Bryant, Recent advances in the theory of holonomy, Astérisque No. 266 (2000), Exp. No. 861, 5, 351-374.

[Del70] P. Deligne, Équations différentielles à points singuliers réguliers, Lecture Notes in Math., 163, Springer, Berlin, 1970.

[GH94] P. Griffiths and J. Harris, Principles of algebraic geometry, Reprint of the 1978 original, Wiley, New York, 1994. 
[GT05] R. M. Guralnick and P. H. Tiep, Decompositions of Small Tensor Powers and Larsen's Conjecture, arXiv:math.GR/0502080, 2005.

[GT06] Symmetric powers and a problems of Kollár and Larsen, preprint, 2006.

[HL97] D. Huybrechts and M. Lehn, The geometry of moduli spaces of sheaves, Vieweg, Braunschweig, 1997.

[Kaw81] Y. Kawamata, Characterization of abelian varieties, Compositio Math. 43 (1981), no. 2, 253-276

[KN96] S. Kobayashi and K. Nomizu, Foundations of differential geometry. Wiley Classics Library, John Wiley \& Sons Inc., New York, 1996.

[Kob86] S. Kobayashi, Homogeneous vector bundles and stability, Nagoya Math. J. 101 (1986), 37-54

[Mil80] J. S. Milne, Étale cohomology, Princeton Univ. Press, Princeton, N.J., 1980.

[MR82] V. B. Mehta and A. Ramanathan, Semistable sheaves on projective varieties and their restriction to curves, Math. Ann. 258 (1981/82), no. 3, 213-224.

[MS80] V. B. Mehta and C. S. Seshadri, Moduli of vector bundles on curves with parabolic structures, Math. Ann. 248 (1980), no. 3, 205-239.

[MY92] M. Maruyama and K. Yokogawa, Moduli of parabolic stable sheaves, Math. Ann. 293 (1992), no. 1, 77-99.

[NS65] M. S. Narasimhan and C. S. Seshadri, Stable and unitary vector bundles on a compact Riemann surface, Ann. of Math. (2) 82 (1965), 540-567.

[Ram66] S. Ramanan, Holomorphic vector bundles on homogeneous spaces, Topology 5 (1966), 159-177.

[Ric72] R. W. Richardson, Jr., Deformations of Lie subgroups and the variation of isotropy subgroups, Acta Math. 129 (1972), 35-73.

[Sei87] G. M. Seitz, The maximal subgroups of classical algebraic groups, Mem. Amer. Math. Soc. 67 (1987), no. 365, iv+286 pp.

[Ses70] C. S. Seshadri, Moduli of $\pi$-vector bundles over an algebraic curve, in Questions on Algebraic Varieties (C.I.M.E., III Ciclo, Varenna, 1969), 139-260, Ed. Cremonese, Rome, 1970.

[Tan38] T. Tannaka, Über den Dualitätsatz der nichtkommutativen topolgischen Gruppen, Tôhoku Math. J. 45 (1938), 1-12.

[Ume78] H. Umemura, On a theorem of Ramanan, Nagoya Math. J. 69 (1978), 131-138

[Yok95] K. Yokogawa, Infinitesimal deformation of parabolic Higgs sheaves, Internat. J. Math. 6 (1995), no. 1, 125-148. 